\title{
BMJ Open The effects of prenatal cannabis exposure on fetal development and pregnancy outcomes: a protocol
}

\author{
Jayleen K L Gunn, ${ }^{1}$ Cecilia B Rosales, ${ }^{2}$ Katherine E Center, ${ }^{3}$ Annabelle V Nuñez, ${ }^{4}$ \\ Steven J Gibson, ${ }^{5}$ John E Ehiri ${ }^{5}$
}

To cite: Gunn JKL, Rosales CB, Center KE, et al. The effects of prenatal cannabis exposure on fetal development and pregnancy outcomes: a protocol. BMJ Open 2015;5:e007227. doi:10.1136/bmjopen-2014007227

- Prepublication history and additional material is available. To view please visit the journal (http://dx.doi.org/ 10.1136/bmjopen-2014007227).

Received 19 November 2014 Revised 29 January 2015 Accepted 3 February 2015

CrossMark

For numbered affiliations see end of article.

Correspondence to Professor John Ehiri; jehiri@email.arizona.edu

\section{ABSTRACT}

Introduction: The effects of exposure to marijuana in utero on fetal development are not clear. Given that the recent legislation on cannabis in the US is likely to result in increased use, there is a need to assess the effects of prenatal cannabis exposure on fetal development and pregnancy outcomes. The objective of this review is to assess the effects of prenatal exposure to cannabis on pregnancy outcomes (including maternal and child outcomes).

Methods and analyses: Major databases will be searched from inception to the latest issue, with the aim of identifying studies that reported the effects of prenatal exposure to cannabis on fetal development and pregnancy outcomes. Two investigators will independently review all titles and abstracts to identify potential articles. Discrepancies will be resolved by repeated review, discussion and consensus. Study quality assessment will be undertaken, using standard protocols. To qualify for inclusion, studies must report at least one maternal or neonatal outcome post partum. Cross-sectional, case-control, cohort and randomised controlled trials published in English will be included. In order to rule out the effects of other drugs that may affect fetal development and pregnancy outcomes, studies will only be included if they report outcomes of prenatal exposure to cannabis while excluding other illicit substances. Data from eligible studies will be extracted, and data analysis will include a systematic review and critical appraisal of evidence, and meta-analysis if data permit. Meta-analysis will be conducted if three or more studies report comparable statistics on the same outcome.

Ethics and dissemination: The review which will result from this protocol has not already been conducted. Preparation of the review will follow the procedures stated in this protocol, and will adhere to the Preferred Reporting Items for Systematic Reviews and Meta-Analyses (PRISMA) guidelines. Ethical approval of data will not be required since the review will use data that are already available in the public domain through published articles and other reports.

\section{INTRODUCTION}

\section{Background}

In 2010, cannabis was used by 129-190 million people worldwide. ${ }^{1}$ In the same year, roughly 13.1 million people were dependent on cannabis. ${ }^{2}$ Early studies found cannabis users to be average people who describe cannabis as a source of pleasure. ${ }^{3-8}$ The reported pleasurable effects include feeling happy, silly, euphoric, relaxed, hedonistic, sensual and foolish. ${ }^{5}$ The most commonly reported negative effects of cannabis use are anxiety, panic and psychotic symptoms. ${ }^{9}$ Other likely adverse effects include impaired psychosocial development in adolescents who regularly use cannabis, diminished respiratory function and cardiovascular disease. ${ }^{10}$ Additionally, laboratory experiments that vary the dose of tetrahydrocannabinol (THC), the chemical compound responsible for the effects of cannabis, find dose-related deficiencies in attention, reaction time, information processing, perceptual coordination and motor performance. ${ }^{10}$ These deficiencies result in increased risk of motor vehicle accidents by cannabis users. $^{10}$

Recreational cannabis users report using cannabis primarily for relaxation, to relieve stress, ${ }^{11}$ and during social activities, ${ }^{12}$ including a variety of leisure activities, such as eating, sex, socialising at the local pub, parties, weddings, funerals, sporting events, listening to music and watching movies and television. ${ }^{5}{ }^{13-15}$ Overall, recreational users report using cannabis while participating in other activities for two different effects, improved relaxation and improved concentration. ${ }^{16}$ Cannabis was also used to make everyday tasks, such as household chores, more tolerable. ${ }^{17}$ Adult, middle-class and employed recreational cannabis users tend to restrict cannabis use to their recreational time and use it to enhance leisure activities and manage life's challenges. ${ }^{16}$ In comparison, those who use cannabis more heavily as a means of escape tend to be poor and socially marginalised. ${ }^{18} 19$ These users have a higher risk of developing serious drug problems. ${ }^{18} 19$ 
Cannabis dependence is higher in males and young adults (20-24 years), although it is not related to increased mortality. ${ }^{2}$

\section{Public health significance of cannabis use during pregnancy}

No increased risk of birth defects has been found in infants exposed to cannabis in utero. ${ }^{102021}$ Other effects of prenatal cannabis exposure on infants, however, are not so clear. The most common outcome linked to cannabis exposure in utero is decreased birth weight. ${ }^{22}$ One study of 7452 infants-including infants exposed to cannabis only in early pregnancy, infants exposed to cannabis throughout pregnancy, non-exposed and tobaccoexposed infants-indicated that birth weight in cannabis exposed infants was $277 \mathrm{~g}$ lower on average compared with those non-exposed to cannabis throughout pregnancy and $156 \mathrm{~g}$ lower for infants exposed to cannabis in early pregnancy only. ${ }^{23}$ Similarly, a study of 1690 infants found that birth weight was, on average, $139 \mathrm{~g}$ lower for infants exposed to cannabis three or more times per week in utero. ${ }^{21}$ Likewise, a third study found a more than twofold increased risk of a low birthweight or small-for-gestational-age infant for those exposed to cannabis 2-3 times a month or more in utero. ${ }^{24}$ However, in this study, the relationship was observed only among European-American mothers, but not in mothers of different ethnicities. ${ }^{24}$

One meta-analysis established that frequent cannabis use (4 or more times a week) was related to a decrease in mean birth weight by $131 \mathrm{~g}$ (95\% CI 52 to $209 \mathrm{~g}$ ). However, the overall pooled estimate of low birth weights OR was not significant $(\mathrm{OR}=1.09 ; 95 \%$ CI 0.94 to 1.27$) .^{25}$ Most studies of the effects of fetal cannabis exposure measure exposure through self-reports by mothers; however, one study compared self-report of cannabis exposure with testing mother's urine. This study found lower birth weight for infants exposed to cannabis when exposure was measured with urine testing, but not when exposure was self-reported. ${ }^{21}$

Other differences have been found for infants exposed to cannabis in utero compared with those non-exposed, including decreased birth length $(0.5 \mathrm{~cm} \text { shorter })^{21} 26$ and decreased gestational length. ${ }^{27}{ }^{28}$ Researchers have examined cerebellar and vascular development in fetuses exposed to cannabis in utero as compared with nonexposed fetuses. While head circumference was reduced in cannabis-exposed fetuses compared with tobaccoexposed and non-exposed fetuses, transcerebellar diameter did not differ. ${ }^{29}$ This suggests that the brains of cannabis-exposed fetuses are no smaller than nonexposed fetuses. ${ }^{30}$ When using a pulsed wave Doppler, prenatal cannabis exposure was associated with an increased fetal pulsatility index and resistance index of the uterine artery. ${ }^{29}$ These may explain any fetal growth deficits in cannabis-exposed infants. ${ }^{30}$ Prenatal cannabis exposure was also associated with a smaller inner diameter of the aorta. ${ }^{29}$ These changes in fetal circulation of cannabis-exposed fetuses were also found in fetuses exposed to tobacco; therefore, these results do not support a cannabis-only exposure effect. ${ }^{30}$ However, the differences between those infants exposed to cannabis and those non-exposed are inconsistent with several studies reporting no differences in birth weight, ${ }^{22} 2731$ head circumference, ${ }^{22} 2632$ birth length ${ }^{22}$ or gestational length. $^{31}$

Studies evaluating the risk of behavioural differences in infants exposed to cannabis during pregnancy also have mixed results. EEG recordings during the first 2 days of life showed subtle differences in sleep patterns between prenatally cannabis-exposed infants and non-exposed infants. ${ }^{32}$ Also, mild delays, measured with the Brazelton Neonatal Behavioral Assessment Scale (NBAS), have been described in infants exposed to cannabis in utero, including increased tremor and startle, and delays in the visual system shortly after birth. ${ }^{32}{ }^{33}$ However, these differences were not seen at 1 month. ${ }^{33}$ Furthermore, another study that assessed neonatal behaviour using the NBAS found no association with cannabis exposure in utero. ${ }^{26}$

\section{Why it is important to do this review}

Overall, the effects of exposure to cannabis in utero on infant growth and behaviour are not clear. Perhaps temporary effects of pain medication during delivery, or of delivery method, may be present when neonates are assessed. It is also possible that these studies are overcome by an inability to control for potential confounding factors. ${ }^{10}$ Women who use cannabis during pregnancy are also more likely to use tobacco, alcohol and other illicit drugs during pregnancy than non-users. ${ }^{34}$ These women also tend to have inferior nutrition than non-users. ${ }^{20}$ Studies that control for potential confounding factors are needed to accurately assess the effects of prenatal cannabis exposure on neonatal behaviour. This systematic review and meta-analysis will critically assess the effects of prenatal cannabis exposure on women and their neonates with the aim of eliciting a clearer evidence of effects of prenatal cannabis exposure on fetal development and pregnancy outcomes.

\section{Objectives}

To assess and critically summarise evidence regarding the effects of prenatal cannabis exposure on fetal development and pregnancy outcomes.

\section{METHODS AND ANALYSES}

The literature search and data collection are currently ongoing. Eligible articles have not been selected and data have not been extracted for the review. The review is anticipated to be completed before the summer of 2015 .

\section{Studies}

Studies must report maternal or neonatal outcomes for women who used cannabis during pregnancy. Only maternal and neonatal outcomes assessed up to 6 weeks 
after birth will be included in this review. Studies included in this review must be: cross-sectional, casecontrol, randomised controlled trials or cohort studies. Studies will not be restricted by date, maternal age, geographical location or by publication status. However, owing to the large volume of research on this topic, only studies published in English will be included. In order to rule out effects of other drugs that may or may not affect neonatal outcomes (eg, cocaine, methadone), studies will only be included if they report outcomes of prenatal cannabis use while excluding other illicit substance. Studies reporting outcomes of prenatal cannabis exposure and use of tobacco and alcohol will be included.

\section{Outcomes}

Studies must report at least one of the following outcomes to be included:

- Maternal

- Pre-eclampsia, eclampsia, postpartum depression, spontaneous delivery, retained placenta, abruption placenta, placenta accrete, placenta praevia, postpartum haemorrhage, anaemia, uterine inversion, uterine rupture, vasa praevia, oligohydramnios, polyhydramnios, maternal mortality, morning sickness, neonatal nursing, abnormal labour and prenatal care.

- Fetal/neonatal

- Preterm delivery, intrauterine growth retardation, head circumference, infant birth weight, low birth weight (under $2500 \mathrm{~g}$ ), gestational age, fetal length, fetal movement, fetal organ maturity, fetal viability, APGAR, neonatal intensive care unit or intensive care unit stay, days in the hospital, reported neonatal problem such as distress, jaundice, spontaneous abortion, neonatal mortality and resuscitation.

\section{Search methods for identification of studies}

We will search the following databases from inception to the latest issue: Ovid, Pubmed, CINAHL, Embase, PsychINFO, Web of Science and Sociological Abstracts. The search criteria can be found in online supplementary appendix A.

By making every effort to contact authors of all included studies to identify whether they have other unpublished or ongoing studies that would meet our inclusion criteria, we will try to ensure that all relevant studies are included. Furthermore, we will read through the list of references in each identified article, and follow-up references that may qualify for inclusion in the review.

\section{Data collection and analysis}

Selection of studies

The initial output of the database searches will be screened independently by two authors to identify and select potentially relevant articles. Titles and abstracts will be reviewed first and duplicates will be removed. Those studies that meet the inclusion criteria will be further explored. The two authors will obtain full copies of these articles and assess them independently to determine which ones meet the predetermined inclusion criteria. If the two authors cannot agree whether to include the article, another author will be asked to review the article in question. Reasons for exclusion will be documented.

\section{Data extraction and management}

Data from eligible studies will be extracted and compared independently by two authors, using a standard data abstraction form. The following data will be extracted from each study:

- Methods

- Study duration and design; study setting; method of cannabis measurement and potential confounders.

- Participants

- Recruitment methods, including inclusion/exclusion criteria; maternal age; neonate age at assessment; race/ethnicity; and other recorded characteristics of participants.

- Outcomes of interest

- All values and SDs for all outcomes of interest related to either the infant or the mother will be extracted, along with adjustments made. We expect outcomes to be reported in a number of ways. For data presented as exact counts or percentages (eg, number of low birthweight infants), we will extract the number presented. These numbers will be transformed in crude ORs. We also expect variables to be presented in an OR form; therefore, we will extract the most adjusted OR as well as the SE. For data presented as a continuous variable (eg, birth weight of infants), we will extract the mean and SD.

\section{Dealing with missing data}

Missing data will be requested from the authors. If missing data are not obtained, that will be noted and the data will be excluded from any further analyses and papers.

\section{Assessment of risk of bias in included studies}

Two authors will assess independently the quality of selected studies using predetermined quality assessment criteria. For cross-sectional studies, the National Collaborating Centre for Environmental Health's tool Critical appraisal of cross-sectional studies will be used to assess the risk of bias. ${ }^{35}$ This tool includes the assessment of study content, method of determining exposure status, comparability of the exposed versus non-exposed group, validation of outcome measures and generalisability. For randomised controlled trials, the Cochrane Collaboration tool will be used to assess the risk of bias. ${ }^{36}$ This tool includes assessments of methods used to randomise participants, allocation concealment, methods used for blinding the participants and the researcher, and methods used for dealing with incomplete data (number lost to follow-up, reasons for drop out). For cohort studies, the Critical Appraisal Skills Programme's Making sense of 
evidence will be used to assess the risk of bias. ${ }^{37}$ This tool includes an assessment of recruitment procedures, measurement of exposure, confounding factors, study results and generalisability. For case-control studies, the Critical Appraisal Skills Programme's Making sense of evidence will be used to assess the risk of bias. ${ }^{38}$ This tool uses three broad categories to address study validity, methodology, recruitment styles and appropriateness, validation of exposure status and measurements, confounding variables and study outcome.

\section{Measures of treatment effect}

Data will be analysed using Review Manager (RevMan) V.5.3. ${ }^{39}$ Basic characteristics of the studies and their results will be included. The data will be analysed by using a randomised and a fixed effect model. Meta-analysis will be conducted if three or more studies report comparable statistics on the same outcome. Data will be pooled using the most adjusted statistics.

\section{Assessment of heterogeneity}

Heterogeneity may be present in different studies. If heterogeneity is present, a meta-regression will be completed to assess which covariates may be the possible source of heterogeneity. If these covariates appear to be significant, subgroup analyses will be presented. The fixed effect model will be used when the level of heterogeneity is deemed acceptable (ie, $\mathrm{p}>0.10$, or $\mathrm{p} \leq 0.10$ and $\mathrm{I}^{2} \leq 50 \%$ ). If the heterogeneity is not acceptable (ie, $\mathrm{p} \leq 0.10$, but $\mathrm{I}^{2}>50 \%$ ), the random effects model will be used.

\section{Data synthesis}

For dichotomous data, an OR will be calculated along with $95 \%$ CIs. An OR will be used to estimate the risk ratio for all studies. For continuous data with outcome measures on a similar scale, a weighted mean difference and $95 \%$ CI will be used in the analysis. In order to compare studies that use different rating scales to assess similar outcomes, a standard mean difference will be calculated. The most adjusted measure of association will always be used.

\section{Sensitivity analysis}

A sensitivity analysis will be run to determine if the results from the study change when studies at risk for bias are included in the study as compared with when they are excluded. If the sensitivity analysis does change the results and a different conclusion may be drawn, caution in interpreting the results and drawing conclusions will be discussed. If no difference is seen, the results will be presented with all studies; the paper will include information about the sensitivity analysis.

\section{ETHICS AND DISSEMINATION}

The review which will result from this protocol has not already been conducted. Literature search and data collection are currently ongoing. Eligible articles have not been selected and data have not been extracted for the review. Preparation of the review will follow the procedures stated in this protocol, and will adhere to the Preferred Reporting Items for Systematic Reviews and Meta-Analyses (PRISMA) guidelines. Ethical approval of data will not be required since the review will use data that are already available in the public domain through published articles and other reports. We anticipate that the review will be completed before the summer of 2015 and be submitted to BMJ Open for consideration.

\section{Author affiliations}

${ }^{1}$ Department of Epidemiology and Biostatistics, Mel \& Enid Zuckerman College of Public Health, University of Arizona, Tucson, Arizona, USA ${ }^{2}$ Department of Community, Environment and Policy, Mel \& Enid Zuckerman College of Public Health, University of Arizona, Phoenix, Arizona, USA

${ }^{3}$ Department of Obstetrics and Gynecology, University of Arizona College of Medicine, Tucson, Arizona, College of Medicine , USA

${ }^{4}$ Arizona Health Sciences Library, Mel \& Enid Zuckerman College of Public Health, University of Arizona, Tucson, Arizona, USA

${ }^{5}$ Department of Health Promotion Sciences, Mel \& Enid Zuckerman College of Public Health, University of Arizona, Tucson, Arizona, USA

Contributors CBR and JEE are the principal investigators. They conceived the original idea for the study and oversaw all aspects of protocol development. JKLG and AVN made substantial contributions to the conception and design of the project. SJG and KEC assisted in the logistics of data collection. JKLG and KEC prepared the first draft of the protocol. All authors reviewed and provided comments on the draft protocol, read and gave approval for release of the final manuscript.

Funding Financial support for this work was provided by the Arizona Department of Health Services, Phoenix, Arizona, USA

Competing interests None.

Provenance and peer review Not commissioned; externally peer reviewed.

Open Access This is an Open Access article distributed in accordance with the Creative Commons Attribution Non Commercial (CC BY-NC 4.0) license, which permits others to distribute, remix, adapt, build upon this work noncommercially, and license their derivative works on different terms, provided the original work is properly cited and the use is non-commercial. See: http:// creativecommons.org/licenses/by-nc/4.0/

\section{REFERENCES}

1. United Nations Office on Drugs, \& Crime. World drug report 2010. United Nations Publications, 2010.

2. Degenhardt L, Ferrari AJ, Calabria B, et al. The global epidemiology and contribution of cannabis use and dependence to the global burden of disease: results from the GBD 2010 Study. PLOS ONE 2013;8:e76635.

3. Becker HS. Becoming a cannabis user. Am J Sociol 1953;59:235-42.

4. Becker HS. Cannabis use and social control. Soc Probl 1955;3:35-44.

5. Goode E. The cannabis smokers. New York: Basic Books, 1970

6. Hallstone M. Updating Howard Becker's theory of using cannabis for pleasure. Contemp Drug Probl 2002;29:821-46.

7. Plant M. Drug-takers in an English town. Br J Criminol 1975;15:181-6.

8. Hirsch M, Conforti R, Graney C. The use of cannabis for pleasure: a replication of Howard S. Becker's study of cannabis use. J Soc Behav Pers 1990;5:497-510.

9. Hall WD, Pacula RL. Cannabis use and dependence: public health and public policy. Cambridge: Cambridge University Press, 2003.

10. Hall W, Degenhardt L. Adverse health effects of non-medical cannabis use. Lancet 2009;374:1383-91.

11. Hathaway A. Cannabis and lifestyle: exploring tolerable deviance. Deviant Behav 1997;18:213-32.

12. Erickson PG. Living with prohibition: regular cannabis users, legal sanctions, and informal controls. Int J Addict 1989;23:175-88. 
13. Hathaway A. Cannabis effects and dependency concerns in long-term frequent users: a missing piece of the public health puzzle. Addict Res Theory 2003;11:441-58.

14. Pearson G. Normal drug use: ethnographic fieldwork among an adult network of recreational drug users in inner London. Subst Use Misuse 2001;36:167-200.

15. Weller R, Halikas J. Cannabis use and sexual behavior. J Sex Res 1984;20:186-93.

16. Osborne GB, Fogel C. Understanding the motivations for recreational cannabis use among adult Canadians 1. Subst Use Misuse 2008;43:539-72.

17. Shukla RK. Using cannabis in adulthood: the experience of a sample of users in Oklahoma City. J Ethn Subst Abuse 2005;4:153-81.

18. Peele S, Brodsky A. The truth about addiction and recovery. New York: Simon \& Schuster, 1991

19. Zimmer L, Morgan JP. Cannabis myths, cannabis facts: a review of the scientific evidence. New York: The Lindesmith Center, 1997.

20. Tennes K, Aritable N, Blackard C, et al. Marihuana: prenatal and postnatal exposure in the human. In: Pinkert T. ed. Current research on the consequences of maternal drug abuse. Rockville, MD: US Department of Health and Human Services, 1985:48-60.

21. Zuckerman B, Frank DA, Hingson R, et al. Effects of maternal cannabis and cocaine use on fetal growth. $N$ Engl $J$ Med 1989;320:762-8

22. Fergusson DM, Horwood LJ, Northstone K. Maternal use of cannabis and pregnancy outcome. BJOG 2002;109:21-7.

23. El Marroun $\mathrm{H}$, Tiemeier $\mathrm{H}$, Steegers EA, et al. Intrauterine cannabis exposure affects fetal growth trajectories: the Generation R Study. J Am Acad Child Adolesc Psychiatry 2009;48:1173-81.

24. Hatch EE, Bracken MB. Effect of cannabis use in pregnancy on fetal growth. Am J Epidemiol 1986;124:986-93.

25. English D, Hulse G, Milne E, et al. Maternal cannabis use and birth weight: a meta-analysis. Addiction 1997;92:1553-60.

26. Day NL, Richardson GA. Prenatal cannabis use: epidemiology, methodologic issues, and infant outcome. Clin Perinatol 1991;18:77-91.

27. Fried PA, Watkinson B, Willan A. Cannabis use during pregnancy and decreased length of gestation. Am J Obstet Gynecol 1984;150:23-7.
28. Gibson GT, Baghurst PA, Colley DP. Maternal alcohol, tobacco and cannabis consumption and the outcome of pregnancy. Aust $N Z J$ Obstet Gynaecol 1983;23:15-19.

29. El Marroun $\mathrm{H}$, Tiemeier $\mathrm{H}$, Steegers $\mathrm{E}$, et al. A prospective study on intrauterine cannabis exposure and fetal blood flow. Early Hum Dev 2010;86:231-6.

30. Huizink AC, Prenatal cannabis exposure and infant outcomes: overview of studies. Prog Neuropsychopharmacol Biol Psychiatry 2014;52:45-52.

31. Linn S, Schoenbaum SC, Monson RR, et al. The association of cannabis use with outcome of pregnancy. Am J Public Health 1983;73:1161-4.

32. Fried PA. Marihuana use by pregnant-women-neuro-behavioral effects in neonates. Drug Alcohol Depend 1980;6:415-24.

33. Fried PA, Smith AR. A literature review of the consequences of prenatal marihuana exposure: an emerging theme of a deficiency in aspects of executive function. Neurotoxicol Teratol 2001;23:1-11.

34. Eyler FD, Behnke M. Early development of infants exposed to drugs prenatally. Clin Perinatol 1999;26:107-50.

35. National Collaborating Centre for Environmental Health. A primer for evaluating the quality of studies on environmental health. Critical appraisal of cross-sectional studies. August 2011. http://www.ncceh ca/sites/default/files/Critical_Appraisal_Cross-Sectional_Studies_ Aug_2011.pdf (accessed Nov 2014).

36. Higgins JP, Altman DG, Gotzsche PC, et al. The Cochran Collaboration's tool for assessing risk of bias in randomised trials. BMJ 2011;343:d5928.

37. Critical Appraisal Skills Programme (CASP UK). Making sense of evidence: 12 questions to help you make sense of a cohort study [Internet]. 31 May 2013. http://www.casp-uk.net/\#!casp-toolschecklists/c18f8 (accessed Nov 2014).

38. Critical Appraisal Skills Programme (CASP UK). Making sense of evidence: 11 questions to help you make sense of a case control study [Internet]. 31 May 2013. http://www.casp-uk.net/\#!casp-toolschecklists/c18f8 (accessed Nov 2014).

39. The Cochrane Collaboration. Review Manager (RevMan) [Internet] [Computer program]. Version 5.3. 13 June 2014. http://tech. cochrane.org/revman (accessed Nov 2014). 